\title{
Research on Influence of Tracking by Electromagnetic Wave Delay to pass through Different Transmission Line
}

\author{
KOU Mingxin \\ China Satellite Maritime Tracking and Control Department, Jiangyin, 214431, China
}

\begin{abstract}
Keywords: DDT (Digital Data Transmission); BPSK(Binary Phase Shift Keying);Maximal-Ratio Combiner; Phase Calibration; Electromagnetic Wave Delay Correction
\end{abstract}

\begin{abstract}
This text mainly introduced the TT\&C\&DDT down signal maximal-ratio combiner and target tracking voltage of angle error, analysis the influence of tracking by electromagnetic wave delay correction in different transmission line, given improvement viewpoint of BPSK signal tracking system by electromagnetic wave delay correction access with the equipment reality serving as the support.
\end{abstract}

\section{Introduction}

Space systems of measurement and control increases the overall design of integrated monitoring and control data transmission system. Certain carriers Unified Control System is designed to support the integration of monitoring and control data transmission mode of the downlink signal diversity combining success can spin around, but found in the test equipment modification link delay difference has a greater impact on diversity combining performance and even cause the system cannot work properly. Aiming at this problem to analyze research and find the essence of the reasons put forward solutions.

\section{Leads to problems}

Certain unified monitoring and control system uses a single carrier pulse Dual tracking system, left and downward, and the right-handed, left-handed difference, a total of four D-difference signal, the new monitoring and control data transmission integrated design supports single-spin and spin around the work of diversity composition working modes. By zeroing calibration tower drive forward the transformation of the way to build the device RF wireless circuits, integrated measurement and control data transmission mode column calibration phase. In the meantime we found a single left-handed, one-handed operation mode, the device calibration phase normal, telemetry data demodulation is correct, after normal school with equipment tracking, telemetry data accuracy and phase error rates to meet the system design requirements. Appear in a spin around diversity synthesis mode is not working, specific performance: diversity synthesis gain is negative; low bit rate difference loss of lock ring mold; high rate difference die ring can be locked, but poor school with reproducible results, abnormal slope, loaded with school results can not be used from the antenna track.

\section{Principle Introduction}

IF signal tracking system consists of a channel, AGC controls, A / D sampling bandpass, composition and reception channel demodulation and channel difference angle error demodulation section, complete polarization selection, AGC control, carrier tracking, angle error signal demodulation, phase correction, the difference between the slope of the correction algorithms to the data.

Single pulse under dual-channel IF receiver system input signal in the form of:

$$
\begin{gathered}
\sum \mathrm{IF}=\mathrm{A} \times \mathrm{D}(\mathrm{t}) \times \cos (\omega \mathrm{t}) \\
\Delta \mathrm{IF}=\mathrm{A} \times \mathrm{D}(\mathrm{t}) \times[\Delta \mathrm{A} \times \cos (\omega \mathrm{t}+\phi)+\Delta \mathrm{E} \times \sin (\omega \mathrm{t}+\phi)]
\end{gathered}
$$


Receiving sub-module and channel demodulation and channel sent to front-end digital signal in-phase and quadrature mixing to obtain I and Q quadrature baseband signals. Capture unit uses a complex FFT operation on the two orthogonal signals spectral analysis, data extraction code phase and carrier frequency. At the same time, the tracking unit two orthogonal signals are sent to LPF, low pass filtered, then sent to the phase detector, data extraction code and carrier phase error, complete digital phase. After the error signal by the loop filter, correcting the local carrier NCO generated with the input signal with a local frequency reference signal, the completion of the carrier tracking. Design principles shown in Figure 1.

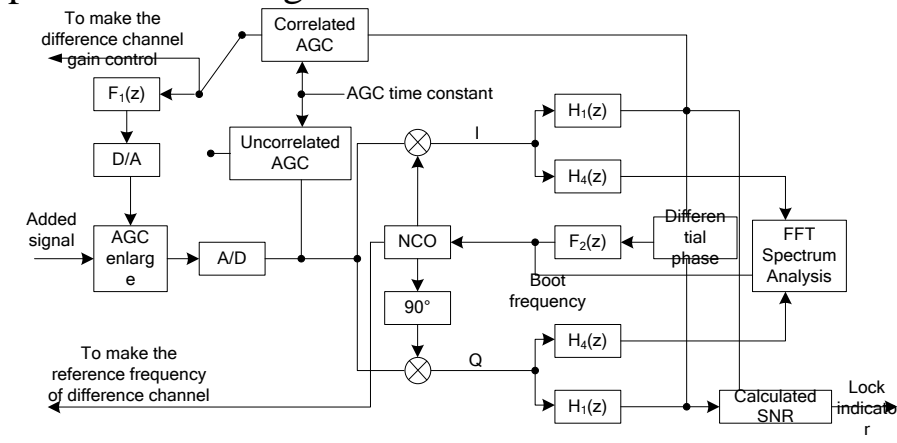

figure 1. Demodulation and channel receiver module schematics

Channel difference angle error demodulation and channel subsystem receives from the local reference code and data carrier signal after the phase correction for mixing associated with the difference signal. Two quadrature output signals I, Q digitally narrowband filtering and slope correction obtained azimuth, elevation error digital component. Design principle shown in Figure 2.

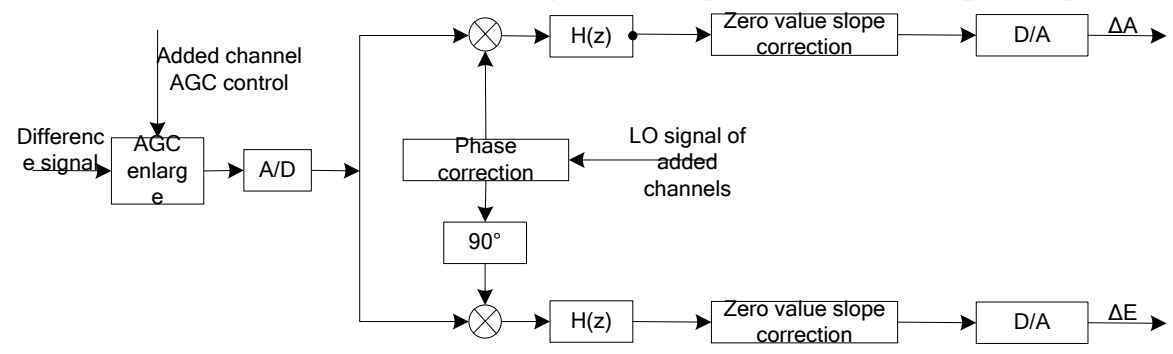

figure 2. Deviation channel demodulation angle error Block Diagram

After school completion phase difference is eliminated and the link phase, and by way of differential signal path signal to data processing, and then adjust the position obtained by the slope, elevation error voltage.

\section{Effects of differential link delay}

Set the system to work in $4 \mathrm{Mbps}$ downlink rate, the use of zero forwarding mode to drive coherent column calibration. Spin around values ranging respectively $1250.748 \mathrm{~m}, 1265.228 \mathrm{~m}$, explain the presence of spin about the delay difference 96.6ns. Labeling terminal baseband device at the entrance to spin around and BPSK signal path delay difference, the difference is the delay measured results 95.2ns, shown in Figure 3, in line with the baseband ranging results, indicating the terminal base with internal two-channel time delay difference is small, when The main difference from the channel extension link. Set the other bit rate, the same test results. 


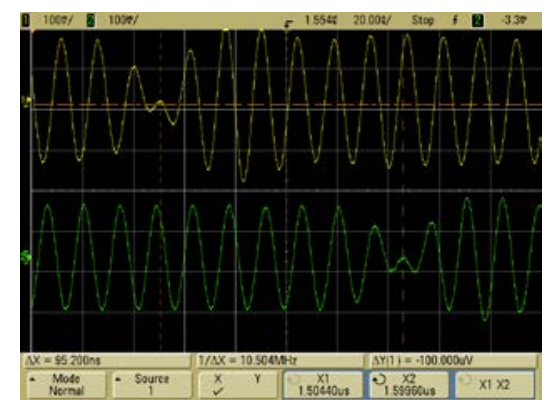

Figure 3. Found spin around and BPSK signals delay difference

\section{Delay Difference on theoretical analysis}

Control data transmission BPSK signal modulated data signal expression after integration system is:

$$
S(n)=\sum_{m=-\infty}^{+\infty} A_{0} g(n-m) \cos \left(\omega_{c} n+\phi_{m}\right)
$$

Although the consolidated monitoring and control system modulation style varied, but essentially nothing more than a modulation signal to control modulation carrier one (or several) parameters, so this parameter modulation signal in accordance with the law and the process of change. Unified carrier system to a sinusoidal signal as a modulated carrier. Demodulated BPSK signal demodulation process is similar to other modulation system signal, the first signal orthogonal decomposition, in-phase and quadrature components; secondly to calculate the instantaneous phase; then sampling the instantaneous phase threshold decision, you can recover data transmission. When the needs of the local carrier demodulation and signal carrier rigorous same frequency and phase, obtained with the same frequency by a digital Costas loop phase.

Phase component:

$$
X_{I}(n)=\sum_{m=-\infty}^{+\infty} A_{0} g(n-m) \cos \phi_{m}
$$

Quadrature components:

$$
X_{Q}(n)=\sum_{m=-\infty}^{+\infty} A_{0} g(n-m) \sin \phi_{m}
$$

Instantaneous phase:

$$
\phi(n)=\sum_{m=-\infty}^{+\infty} g(n-m) \phi_{m}
$$

Under diversity synthesis mode, differential mode loop output Left and right, NCO signal to achieve the Left and right, signal carrier frequency and phase tracking, the system is not designed to delay correction. Synthesis coefficient by spin around signal symbol SNR calculation, since the calculation handedness signal symbol SNR uses the same route symbol timing pulse, and spin around when the signals delay difference is large, the calculated two signals poor signal to noise ratio will increase, resulting in the synthesis of low signal to noise ratio, gain loss synthesis, synthesis of single-noise ratio even lower than the signal to noise ratio, the combined gain is negative, when the signal to noise ratio is less than the difference between the calculated results threshold, the system exits the diversity synthesis, differential mode loop loses lock.

\section{Delay difference affect the measured results}

Table 1 shows the spin around time delay difference 95.2ns, at each rate BPSK diversity combining gain Found circumstances. As can be seen, when the rate of $4 \mathrm{Mbps}$, the difference between the left and right rotation delay rate of $38 \%$, then the signal to noise ratio as low as synthetic telemetry lockout threshold. 
Table 1. BPSK diversity composition gain Found

\begin{tabular}{|c|c|c|c|}
\hline $\begin{array}{c}\text { BPSK data rate } \\
(\mathrm{Mbps})\end{array}$ & $\begin{array}{c}\text { lock of differential } \\
\text { ring }\end{array}$ & $\begin{array}{c}\text { Diversity synthesis } \\
\text { Eb/N0 }\end{array}$ & Single rotation Eb/N0 \\
\hline 0.26 & locked & 30.2 & 34.1 \\
\hline 0.5 & locked & 23.5 & 31.2 \\
\hline 1 & locked & 17.2 & 27.9 \\
\hline 2 & locked & 10.2 & 19.7 \\
\hline 4 & locked & 1.1 & 13.6 \\
\hline 6 & unlocked & - & 10.9 \\
\hline
\end{tabular}

Phase unit in school, and only the difference signal phase correction, carried out without delay correction. After the completion of phase correction difference signals and signals with phase alignment, the time difference n cycles, although not on the difference between the carrier signals normalized tracking. Angle error demodulation unit astray de data processing, the use of the symbol timing pulse signals and, if the rate is higher, and the time delay difference is large, there is to a data processing error, causing the automatic correction phase slope correction means not stable. 4Mbps bit rate, and the difference between the delay phase correction curve 95.2ns burr obvious, many schools with poor consistency, Fig. 4, after the school was unable to stabilize the output orientation phase is complete, the elevation error voltage, as shown in Figure 5.

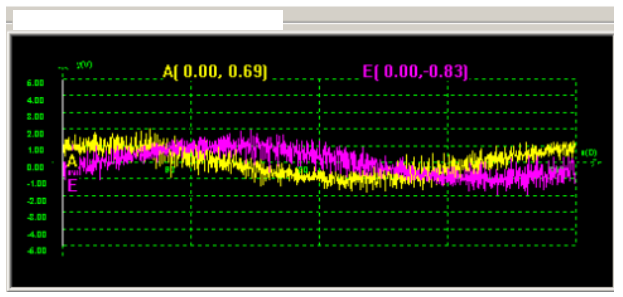

Figure 4. 4 Mbps rate and differential phase delay correction curve 95.2ns
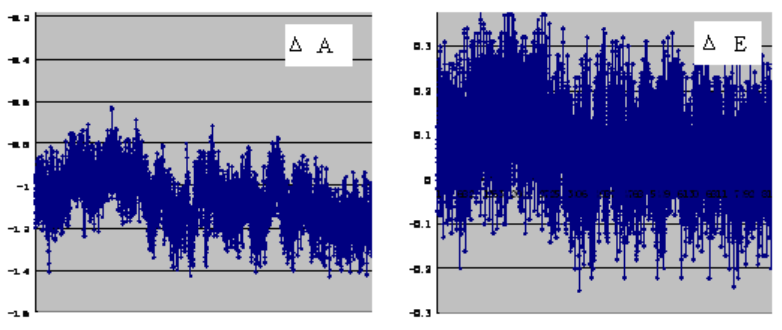

\section{Introduces the delay correction amount of system improvements}

When a system of fixed channel link data symbols delay value is determined, the delay depends on the feed network, is put on the next drive, cables and other electrical characteristics link. So when the system left, right-handed, and the difference channel is fixed, the data link delay difference between symbols is a fixed value, as long as the two links do not change, the value does not change. Measured by the system to validate the delay correction value relatively fixed amount, without the impact rate, by the point of impact at 1 ns frequency magnitude, that dispersion is small. As long as the device link devices did not change after a BPSK by RF wireless signal delay calibration can be used normally, can be combined with the tracking phase calibration performed delay calibration to get this fixed value, to facilitate the tracking receiver diversity combining, and , amplitude and phase difference between the channel normalization process, the correction value left and right-handed, and the difference signal synchronization error.

When the baseband delay the introduction of amendments, the left and right-handed, and the difference link signal delay difference control in less than one symbol, tower of calibration. System works in the collection success rate normal hours and can achieve $2.5 \mathrm{~dB}$ gain; phase correction unit curve smoothing, several schools with good consistency; after loading phase, angle error output stability, good performance measurement system to track improvement. 


\section{Reference}

[1] CCSDS.RADIO FREQUENCY AND MODULATION SYSTEMS[S].401 (5.2) B-2 .2009.

[2] D.M .Dicarl, C L Weber. Statistical performance of single dwell serial synchronization systems. IEEE Transactions on communications, 2010:1382-1388.

[3] SPILLARD C.A serial-parallel FFT corrugators for PN code acquisition from LEO satelites[J]. IEEE Transactions on communications,2008:446-448.

[4] R.A.Iltis. Joint estimation of PN code delay and multipath using the Extended Kalman Filter[J],IEEE Transactions on communications.2010:1677-1685. 